\title{
What is signal and what is noise in the brain?
}

\author{
Andreas Knoblauch ${ }^{a}$ Günther Palm ${ }^{a}$ \\ ${ }^{a}$ Department of Neural Information Processing \\ University of Ulm, Oberer Eselsberg, D-89069 Ulm, Germany \\ email: \{knoblauch,palm\}@neuro.informatik.uni-ulm.de
}

\begin{abstract}
The response of a cortical neuron to a stimulus can show a very large variability when repeatedly stimulated by exactly the same stimulus. This has been quantified in terms of inter-spike-interval (ISI) statistics by several researchers (e.g., Softky and Koch, 1993). The common view is that this variability reflects noisy information processing based on redundant representation in large neuron populations. This view has been challanged by the idea that the apparent noise inherent in brain activity that is not strictly related or temporally coupled to the experiment could be functionally significant. In this work we examine the ISI statistics and discuss these views in a recently published model of interacting cortical areas (Knoblauch and Palm, 2002). From the results of further single neuron simulations we can isolate temporally modulated synaptic input as a main contributor for high ISI variability in our model and possibly in real neurons. In contrast to alternative mechanisms, our model suggests a function of the temporal modulations for short-term binding and segmentation of figures from background. Moreover, we show that temporally modulated inputs lead to ISI statistics which fit better to the neurophysiological data than alternative mechanisms.
\end{abstract}

Key words: neural noise, internal states, ongoing activity, coefficient of variation (CV)

\section{Introduction}

When we talk about "neural coding", we presuppose that we understand what is the "signal" that is represented in the neural response and what is "noise".

$\overline{1}$ We are grateful to Bill Softky for valuable comments. This work was partially supported by the MirrorBot project of the EU. 
This presupposition is certainly not always justified, in particular when we try to interprete recordings from higher vertebrate's cerebral cortex, where a neuron can show a very large variability, for example, when repeatedly stimulated with exactly the same visual stimulus ((Softky and Koch, 1992, 1993; Arieli et al., 1996); (but see Mainen and Sejnowski, 1995; Bair and Koch, 1996)).

The distinction of signal and noise is common in the experimental literature and is usually made and understood with respect to the experimental protocol: neural responses that are related to the experimental protocol are regarded as signals and those that are not related are regarded as noise. When one is dealing with sensory pathways towards (or motor pathways from) the brain, this experimentally motivated definition of signal and noise is likely to coincide with the point of view of the experimental animal, provided the proper (i.e. behaviorally relevant) stimuli are used. But even in this situation, it may happen that the animal is interested in features of the stimulus or the whole experimental situation which are disregarded by the experimenter. In this case the neural responses on the sensory pathway may signal those features which are regarded as "noise" by the experimenter. This problem becomes much more prominent when one tries to understand recordings from cortical areas, even primary sensory areas, because these receive much more input from other (usually "higher") cortical areas than from the sensory pathway.

If one is not aware of (or disregards as unimportant) this distinction between "noise from the experimenter's point of view" and "noise from the animal's point of view", one may be surprised about the apparent noisiness of neural responses and compelled to find particular explanations for this high amount of noise. This tendency can be found in the literature (e.g., Shadlen and Newsome (1998)) leading directly to the idea that the animal's brain may be faced with the same problem as the experimenter to "get rid of " the noise. The experimenter does this by repetitive trials and temporal averaging. The animal does not have so much time, so - the argument goes - it has to do population averaging instead. But the every important entity must be represented in a sufficiently large population of noisy neurons which all convey the same signal (which appears to be a big waste).

We believe that this picture is much too pessimistic and in fact unrealistic, because those neurons do not all carry the same signal. They perhaps do so with respect to the ongoing experiment, but not with respect to what may be behaviorally relevant for the animal. This means, the observed variability could be caused by internal states of the brain possibly related to attention, expectation, motivation, "binding" to other percepts, or generally to the activation of global "cell assemblies" (Hebb, 1949; Palm, 1982, 1990; Knoblauch and Palm, 2002). These internal states could change on a relatively fine time scale (tens to hundreds of milliseconds) and could play an important role in cortical function (e.g. Softky, 1995; Abeles et al., 1995; Kenet et al., 2003; Ringach, 
2003). However, if not locked to stimulus onset they would be invisible in experiments when averaging recordings over many stimulus presentations.

Another, more technical problem concerning expectedly high noise in single neurn firing patterns is the observed high coefficient of variation (CV) of the inter-spike-interval (ISI) distribution (which is defined as the ratio of the standard deviation to the mean of the ISI histogram; cf. Fig. 2c). Real neurons often exhibit a large CV around 1 (Softky and Koch, 1992) which would be expected if the neuron's spike train was a Poisson process. Values above 1 are unlikely if the neuron integrates a large number of independent synaptic inputs.

Several modelling attempts have been made in order to isolate the cause of the high firing variability (for a brief review see Christodoulou and Bugmann, 2001). Candidate causes investigated in previous studies refer either to the spike generation mechanisms or the properties of synaptic inputs that drive the neuron. For example, Troyer and Miller (1997) obtained relatively high $\mathrm{CV}$ values for a high gain (slope of the firing frequency vs. injected current curve) working point of the neuron. Similarly, Bugmann et al. (1997) stressed that high CVs occur in simple leaky integrator models with partial reset on the somatic membrane potential, although very high $C V$ values $(\geq 1)$ seem to have a tendency to be accompanied by bursts which have been excluded from analysis by Softky and Koch (1993).

With respect to synaptic input variability, Softky and Koch (1992) argued that temporal integration of random excitatory spikes is inconsistent with high CV values, while Shadlen and Newsome (1994) showed that relatively high CV values can be obtained if the neurons receive a balance of both excitatory and inhibitory inputs. As further candidates network effects (Lin et al., 1998; Hansel and Sompolinsky, 1996; Usher et al., 1994), temporally correlated inputs (Stevens and Zador, 1998; Feng and Brown, 2000), and long intra-neuron time constants (e.g., NMDA conductances in (La Camara et al., 2002)) have been investigated.

In this work we try to answer the following questions: (i) What causes the variability, and (ii) can we relate this to behaviorally important global brain activity? In section 2.1 we reconsider results from a recently published large neural network model of two interacting cortical areas (Knoblauch and Palm, 2002). The aim of our model was to understand the population activity that leads to local field potentials (LFP) and LFP-correlograms that have been ovserved in visual cortical areas (Engel et al., 2001; Eckhorn, 1999). Re-examining the ISI histograms of single neurons in this model, we find that the neurons exhibit very high CV values (see Fig. 1). Since these values are partially due to bursts and fast oscillations (around $50 \mathrm{~Hz}$ ) we then simulate in section 2.2 simply a single neurons in a non-bursting and non-oscillatory regime but with 
spike trains otherwise exhibiting similar temporal properties as in our network model (see Figs. 1,2). We find not only high CV values similar to those measured for real neurons, but possibly also a better correspondence to physiologically observed CV vs. mean ISI curve encountered in neurons from areas V1 and MT of awake behaving monkey (cf. Fig. 3 vs. Fig. 3 in Softky and Koch (1993)). In section 3 we conclude that the reason for the high CV values in our models can be regarded as a slowly changing temporal covariation of the afferents to the observed neuron. Thus we have found a simple explanation for the data of Softky and Koch (1993) which is consistent with our general ideas presented in the introduction and modelled and discussed in more detail in our previous paper (Knoblauch and Palm, 2002).

\section{Modelling and results}

\subsection{Network model}

Recently we have implemented a large model of two interacting visual cortical areas in order to investigate scene segmentation and "binding" by spike synchronization (Knoblauch and Palm, 2002). Each neuron in this model receives inputs from more than 1000 other model neurons. The detailed connecting is not important for the present analysis; the neuron model is described in the appendix. For more details see Knoblauch and Palm (2002). We have related the results of this model to a number of experimental findings such as synchronization on different time scales, ongoing activity, and attention. Fig. 1a,b shows the recordings from three neurons in a "primary visual area" responding to a triangle stimulus in a scene composed of three overlapping shapes (a triangle, a rectangle, and an ellipse). The three neurons are not directly connected, but receive common input from the other area. We observed on and off "switching" of an enhanced activity state induced by reciprocal connections to an "associative area". The switching was of course induced by the stimulus (the three shapes) that was present for a long time (two seconds), but not temporally linked to the stimulus onset. The local neurons responding to one of the three shapes are simultaneously in the enhanced state for about 50$500 \mathrm{msec}$ until they habituate and another neuron population representing a different shape is activated. This kind of 'activation switching' is also reflected on the single cell level (Fig. 1a,b). However, the switching is not locked to the stimulus (Fig. 1b): When averaging over repeated stimulations, the peristimulus-time-histogram (PSTH) is essentially flat (see Fig. 10 in Knoblauch and Palm, 2001, cf. Fig. 2e,f). Thus, since the activity modulations induced by the "switching" do not covary with the stimulus they may be interpreted as stimulus-irrelevant "noise", although, at least in our model, they are functionally relevant for scene segmentation and binding of distributed features. 


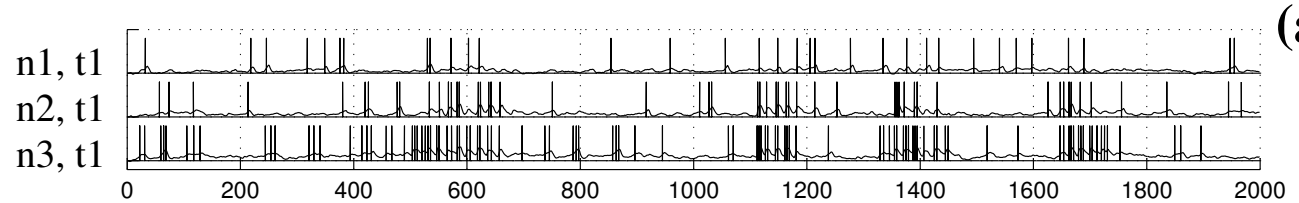

(a)

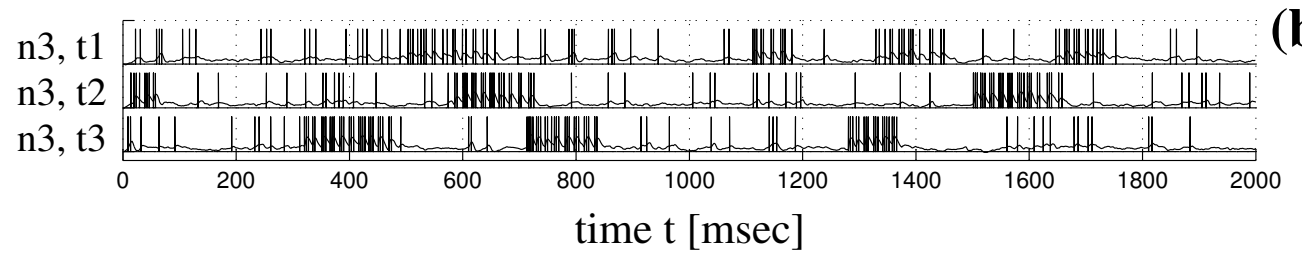

(b)

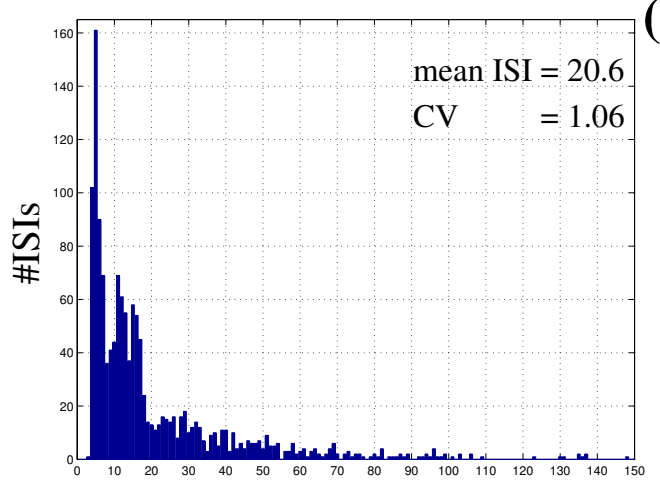

ISI [msec] (c)

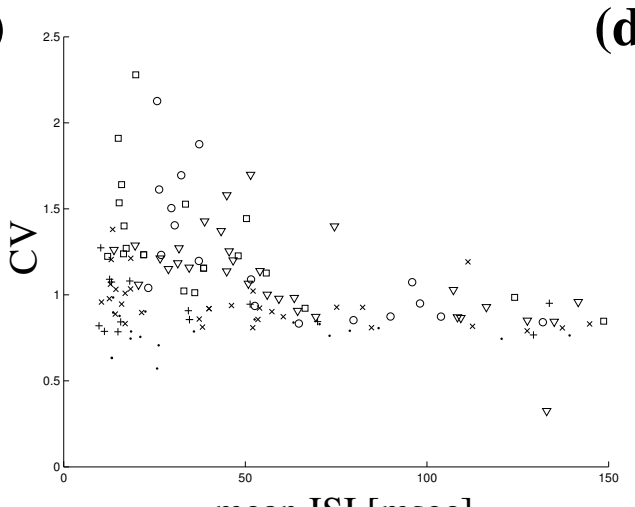

Fig. 1. High CV values for ISIs in a previously published model of two interacting visual cortical areas (Knoblauch and Palm, 2002) stimulating for 2sec with a scene of three overlapping objects (triangle, ellipse, rectangle). a: Simultaneous recording from three neurons (n1-n3) representing the triangle stimulus. The recordings show an on and off switching between two activation states (note increased activity around $\mathrm{t}=600,1150,1400,1700 \mathrm{msec}$ ) occurring simultaneously for neurons coding the same object. In this model, the switching is functionally relevant for scene segmentation. b: Three recording trials (t1-t3) for neuron $n 3$ using the same stimulus as in (a). The switching is not linked to the stimulus onset and therefore not visible when averaged over multiple trials (in a PSTH). c: ISI histogram for neuron $n 3$ exhibiting a high CV value. The gap around $8-10 \mathrm{msec}$ indicates the presence of oscillations and "bursts" (see also the potential oscillations in (a),(b)). d: CV vs mean ISI for different neurons in similar simulations as for (a)-(c), where either a superposition of three shapes (same simulation as in (b); triangle $\triangle$, ellipse $\circ$, rectangle $\square$ ) or single shapes (triangle $\times$, ellipse $\cdot$, rectangle + ) have been used for stimulation. $C V$ : coefficient of variation. ISI: inter-spike-interval. PSTH: peri-stimulus-time-histogram.

This leads us to two goals of this work: First, we want to test whether our model is compatible with the finding of high response-variability, i.e., if single neuron recordings in our model exhibit similarly high CV's as reported in neurophysiological experiments. If so, we secondly want to investigate what causes the high $\mathrm{CV}$ values in our model and perhaps also in real neurons. 
When computing the CVs for spike recordings from our model neurons (Fig. 1a,b) we found very high values usually in the range well above 1 (Fig. 1c,d). It was not easy, however, to dissect the reasons for the high CV in this model since many of the neurons (in particular those with high firing rates) exhibited fast oscillations and bursting (Fig. 1c) which have been excluded from the analysis, for example, by Softky and Koch (1992).

Thus in order to isolate the causes of the high CV values, we simplified our large network model and simulated simply single neurons with input statistics such that the output spike train exhibits similar properties as in our original model, but no fast oscillations or bursts.

\subsection{Single neuron model}

For the neuron we used a standard leaky integrate-and-fire model (see appendix) without any habituation in order to flat PSTHs (cf. Fig. 2b,f). In real experiments, typical PSTHs are not flat, showing on-responses and habituation which are not included in the CV analysis. Actually, these nonstationarities of real neurons are usually eliminated before computing the ISI statistics by normalizing to the instantaneous firing rate (see Softky and Koch (1993)).

The neuron received synaptic inputs from 5000 excitatory and 500 inhibitory presynaptic units modelled as independent Poisson processes with base rates of 1 and 2 spikes/sec, respectively (see appendix for details). We simulated four different input regimes: In the excitatory regime the neuron received only excitatory inputs of different synaptic strengths, in the balanced regime the neuron received both excitatory and inhibitory inputs where we varied the strength of the inhibitory synapses. Finally, in the modulated excitatory and modulated balanced regimes we additionally modulated the strengths of the excitatory inputs by a cosine function with frequency $f=7 \mathrm{~Hz}$ in order to get results similar to our original model (Fig. 1).

Figure 2 compares the balanced regime to the modulated balanced regime for fixed strenghts of excitation and inhibition where the neuron's firing frequency was about the same in both input regimes. For the non-modulated regime the results are comparable to previous work (e.g. Softky and Koch, 1992; Shadlen and Newsome, 1994). The PSTH (Fig. 2b) indicates a stationary response of the neuron during stimulation, and the ISI histogram shows a distribution similar as expected for a Poisson process (Fig. 2c). In contrast to a corresponding Poisson process (which would have an exponential ISI distribution) very small ISIs (due to refractoriness) but also large ISIs are missing, while too many medium ISIs are present (Fig. 2d). 


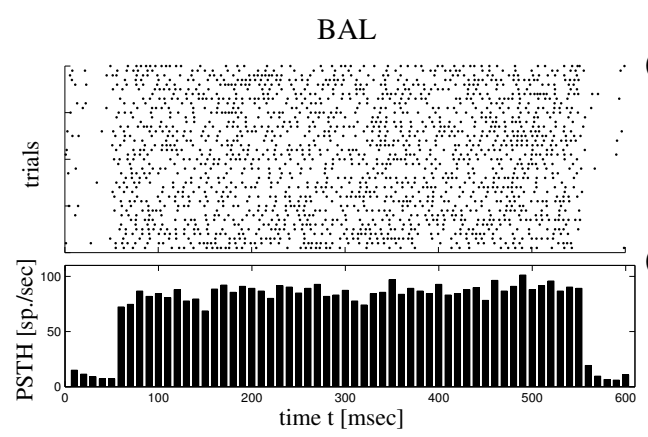

(a)
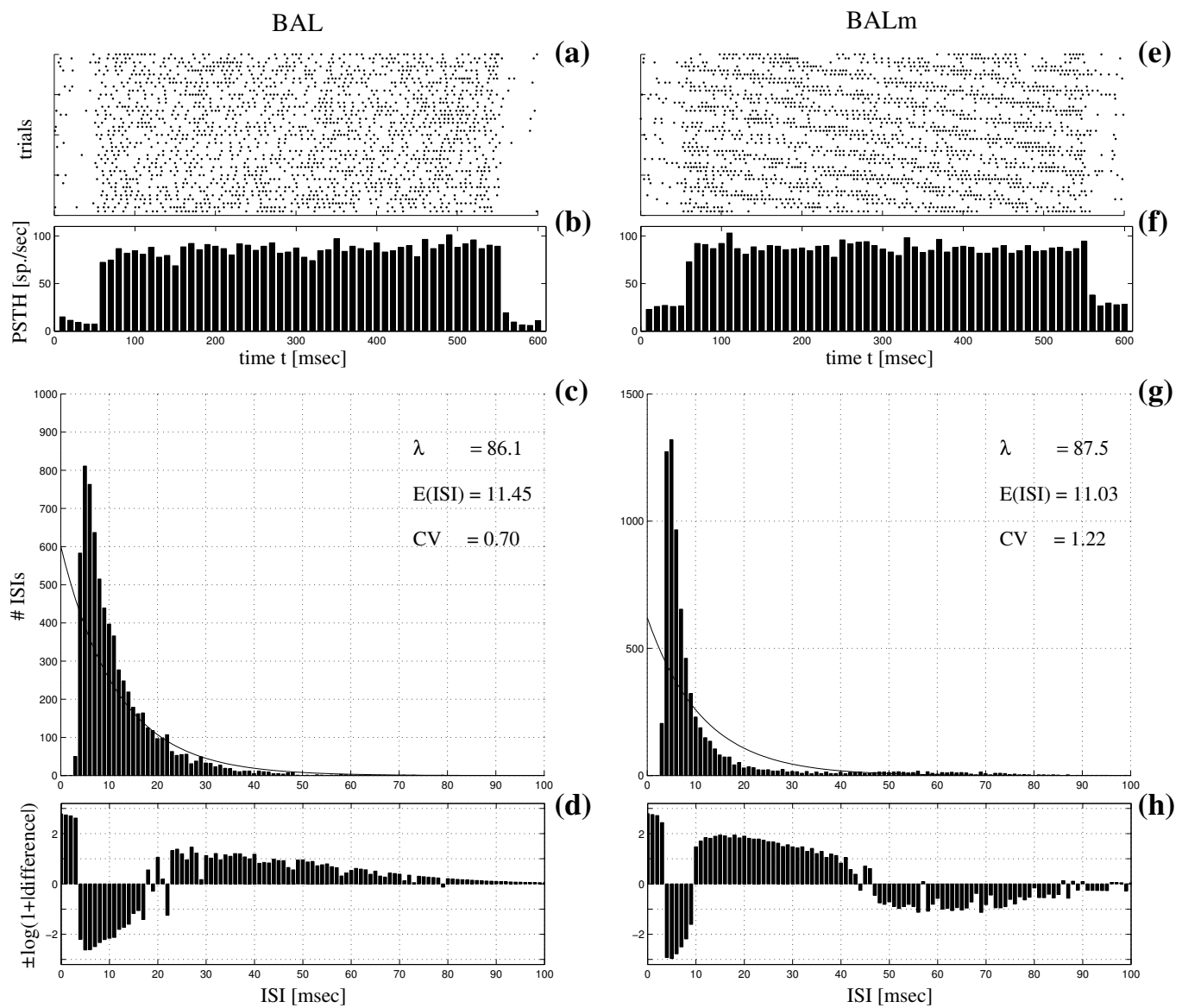

(c)

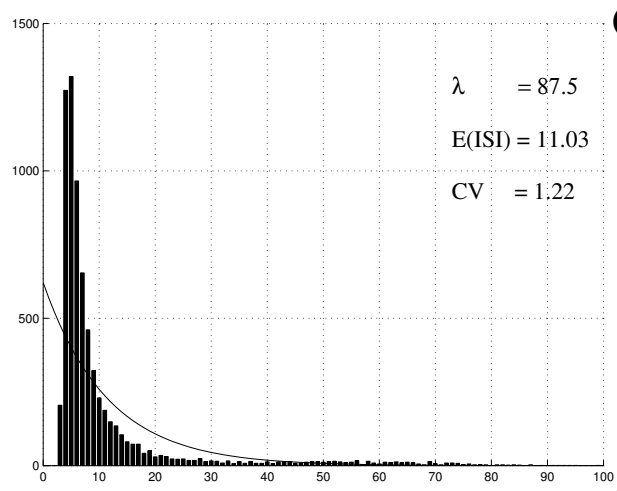

(g)

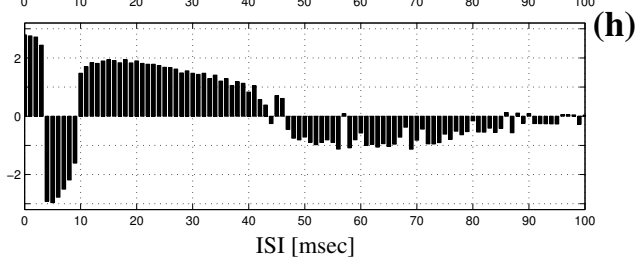

Fig. 2. Comparison of two regimes where responses of a simulated neuron exhibit high variability. Left panels (a-d) show results for balanced excitation and inhibiton (BAL), right panels $(\mathbf{e}-\mathbf{h})$ the results when additionally the input is modulated periodically with $\mathrm{f}=7 \mathrm{~Hz}(\mathrm{BALm})$. a/e : Spike recordings of 40 trials of length $600 \mathrm{msec}$ where the stimulus was active for $500 \mathrm{msec}$ starting with $\mathrm{t}=50 \mathrm{msec}$. Each line corresponds to one trial, each black dot corresponds to a spike. b/f : PSTH (peri-stimulus-spike-histogram) computed from 166 trials. c/g : Histogram (bin size $1 \mathrm{msec}$ ) of the distribution of inter-spike-intervals (ISIs) during stimulation. Numbers indicate mean spike rate $(\lambda)$, mean ISI (E(ISI)), and coefficient of variation (CV) during stimulation. The thin black line corresponds to the (exponential) ISI distribution as expected from a Poisson point process with rate $\lambda . \mathbf{d} / \mathbf{h}:$ Logarithmic plot of the difference $d$ between the ISI histogram and the exponential prediction (see $\mathbf{c} / \mathbf{g})$. The plot $\operatorname{shows} \operatorname{sgn}(d) \cdot \log _{10}(1+|d|)$. Positive values indicate histogram values smaller than expected from the exponential distribution, negative values indicate histogram values larger than expected.

For the modulated input regime the temporal modulations are clearly visible in the stripes of the single trial plot (Fig. 2e). In contrast, the PSTH (Fig. 2f) is essentially flat because the temporal modulation is not locked to stimulus onset. The ISI histogram still looks similar to that of a Poisson process (Fig. $2 \mathrm{~g}$ ) although in contrast to the non-modulated regime it contains too many large ISIs (Fig. 2h). 
Fig. 3 plots the CV vs. mean ISI curve for the four regimes stimulating with different input strengths. As reported earlier (e.g. Softky and Koch, 1992) large CV values cannot be obtained for the excitatory regime. Relatively high $\mathrm{CV}$ values are obtained for the balanced regime where the input consists of both excitatory and inhibitory inputs. But very high CV values are possible if the strength of synaptic inputs are temporally modulated. Note that the curves for both the modulated excitatory and modulated balanced regimes are peaked. Similar peaks resulted from the analysis of real neurons (see Fig. 1 in Softky and Koch (1992), Fig. 3 in Softky and Koch (1993)), although it is not clear if they are artificially caused by the limited range of ISI histograms (see methods in Softky and Koch, 1993).

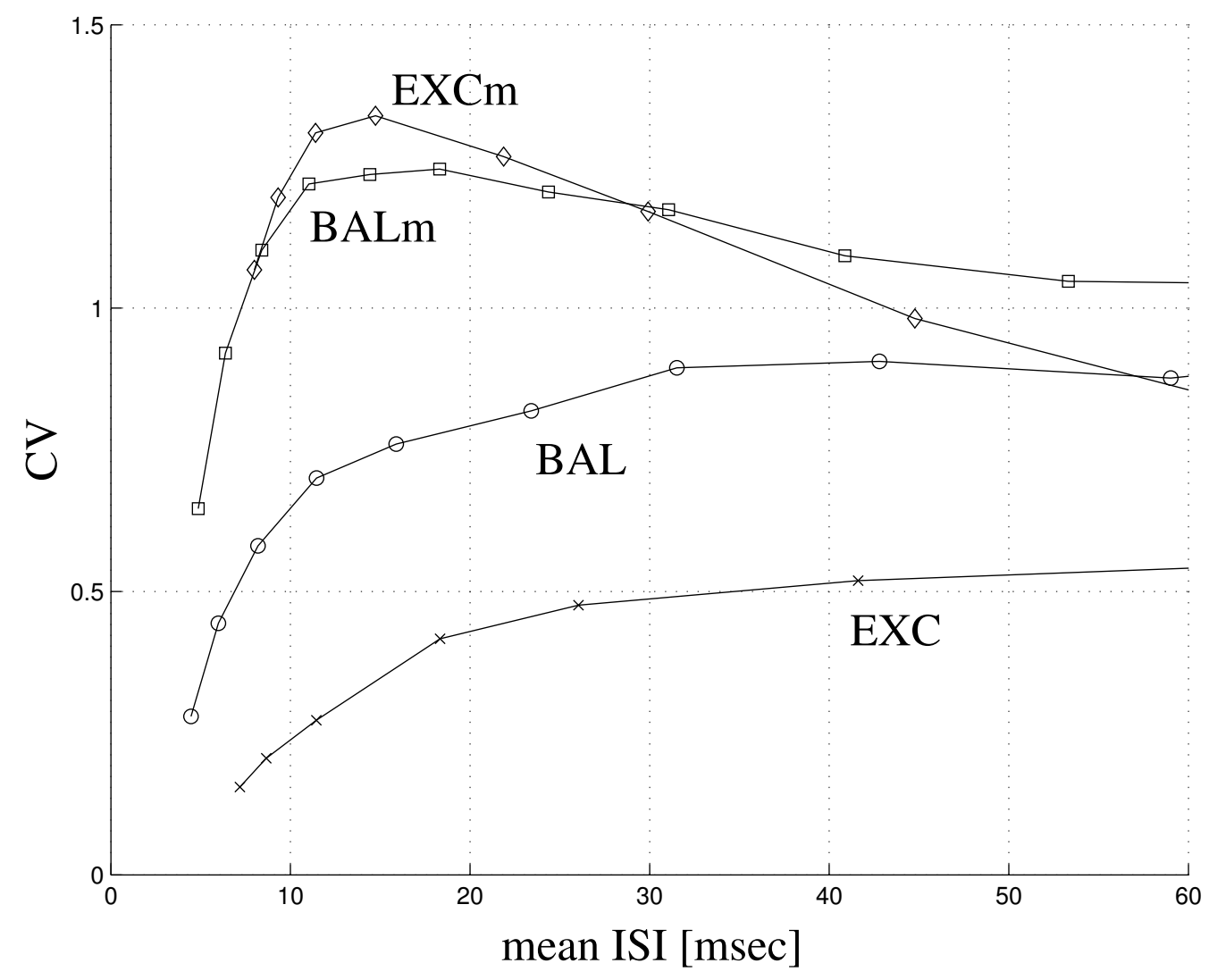

Fig. 3. Comparison of the coefficient of variation (CV) as a function of mean interspike interval (ISI) of a single simulated neuron for four different input regimes. EXC: The neuron receives only excitatory input (of different synaptic strengths). BAL: In the balanced regime the neuron receives both excitatory and inhibitory inputs (the strength of the inhibitory inputs have been varied). EXCm/BALm: Same as EXC/BAL but the input strengths are modulated according to a cosine with frequency $f=7 \mathrm{~Hz}$. High CV values are obtained for the balanced or modulated regimes (BAL, BALm, EXCm). For the modulated regimes (BALm, EXCm) the CV curves are peaked similar as for neurophysiological data (cf. Fig. 1 in Softky and Koch, 1992, but see text).

Further simulations (data not shown) indicate that our qualitative results 
do not depend on fine parameter tuning (see appendix). For example, for modulation frequency between $f=0.5 \mathrm{~Hz}$ and $f=20 \mathrm{~Hz}$ the peaks occur for mean ISIs between $10 \mathrm{msec}$ and $20 \mathrm{msec}$, although the absolute CV values become very large for $f \leq 2 \mathrm{~Hz}$ and quite small for $f \geq 20 \mathrm{~Hz}$. It is also possible to obtain CV values above 1 (but not peaks) for the unmodulated balanced regime, but only for very small $n_{\text {in }} \cdot L_{\text {in }}$ and large $s_{\text {in }}$.

\section{Conclusion}

This work was initiated by discussions with participants at the Neural Coding Workshop about noise and ISI statistics in real neurons in working brains. These triggered the idea to look at the ISI statistics in our recently published model of interacting visual cortical areas designed to investigate spike synchronization and "binding" (see Fig. 1; see Knoblauch and Palm, 2002). In this model, scene segmentation is accomplished by "activation switching" leading to temporal modulations of spike activity on a time scale of $50-500 \mathrm{msec}$, where the modulations are correlated for neurons coding the same object, but not locked to stimulation and therefore not visible in PSTHs.

In the present work we first have shown that many neurons from our previous model exhibit a high variability in their stimulus responses and very high CV values similar to real neurons analyzed in previous studies (e.g. Softky and Koch, 1993). However, in contrast to the real neurons our model neurons were in an oscillatory and sometimes bursting regime (Fig. 1c,d). Thus, in order to isolate the cause of the high firing variability, we then simulated single neurons in an input regime similar to our previous model, but without exhibiting any fast oscillations or bursting (Figs. 2,3). The results indicate that the high CV values can be attributed mainly to the slower temporal modulation of the instantaneous firing rate causing Poisson-like ISI histograms with "long tails" (see Fig. 2e-h). They cannot be generated by fast oscillatory modulations in the $40-60 \mathrm{~Hz}$ range (data not shown).

The most cautious interpretation of our work just suggests that temporally modulated synaptic input is another possible explanation for high firing variability, adding to other known mechanism (see introduction). Indeed, it is most probable that the high CV values of real neurons are caused by a mixture of several such mechanisms, slow temporal input modulation and excitationinhibiton balance being the most prominent ones (cf. Segundo et al. (1998)). However, in contrast to many other models, our previous work on interacting cortical areas postulates a function of the "noisy" within-trial and inter-trial firing variability, since in this model the slow temporal modulations are essential for short-term binding of distributed features and the segmentation of figures from background (Knoblauch and Palm, 2002). Furthermore, the CV 
vs ISI curves of Fig. 3 exhibit peaks specifically for the modulated regimes, which may fit better to the neurophysiological data (although the real neurons' peaks might be caused partially by the limited width of ISI histograms; cf. Fig. 1 in Softky and Koch (1992), Fig. 3 and methods in Softky and Koch (1993)). The absolute CV values for our modulated regimes can be even higher than for most real neurons, but this may be due to the artificially regular and smooth cosine modulation. In contrast in our original model the modulation was much more irregular (cf. Fig. 1). Thus we expect that the corresponding $\mathrm{CV}$ values for our model (however, in regimes without oscillations and bursting) would be somewhere between the curves for the unmodulated and modulated balanced regimes (Fig. 3), i.e. in the range of the experimental data.

Our results illustrate why the interpretation of temporal averaging (over time or trials) as a replacement of spatial averaging (over a population of neurons) - the ergodic hypothesis - may be problematic in the brain. The experimental situation can be improved by looking simultaneously at multiple instead of single units. However, there seems to be currently no way of directly checking this hypothesis by recording from a substantial proportion of all the neurons in one brain area at the same time. So temporal averaging and the display of PSTHs remain indispensable and important methods in neurophysiology. However, one should resist the suggestion that the brain uses the same methods by replacing temporal with spatial averaging. In fact, population averaging and rate coding are pessimistic ideas that tend to underestimate the potential of single neurons for information processing (Softky, 1995).

Information theoretical evaluations of experimental results will generally underestimate the information transmission capacity (transinformation) of neurons or neural populations, because it is measured only with respect to the experimentally accessible variables (cf. Palm, 1996; Borst and Theunissen, 1999). But 'noise' with respect to the experiment may be 'signal' with respect to other circumstances that are interesting or important for the animal.

\section{Appendix: Neuron model and input generation}

For our new simulations we used a single standard leaky integrate-and-fire neuron model that was also used in the previous paper (Knoblauch and Palm, 2002; Knoblauch, 2003). The equation for the membrane potential $x$ as a function of time $t$ is

$$
\tau_{x} \frac{d}{d t} x(t)=-x(t)+g_{\mathrm{ex}}(t)\left(E_{\mathrm{ex}}-x(t)\right)+g_{\mathrm{in}}(t)\left(E_{\mathrm{in}}-x(t)\right),
$$


where $\tau_{x}=10 \mathrm{msec}, E_{\text {ex }}=80 \mathrm{mV}$, and $E_{\text {in }}=-10 \mathrm{mV}$. Excitatory and inhibitory synaptic inputs are integrated by the conductances $g_{\mathrm{ex}}$ and $g_{\mathrm{in}}$, respectively. Each spike arriving at an excitatory (or inhibitory) synapse causes an instantaneous increase of $g_{\text {ex }}$ (or $g_{\text {in }}$ ) by $s_{\text {ex }}$ (or $s_{\text {in }}$ ), followed by an exponential decay with time constant $\tau_{\text {ex }}=5 \mathrm{msec}$ ( or $\tau_{\text {in }}=7 \mathrm{msec}$ ). The neuron emits a spike as soon as $x$ exceeds the threshold $\Theta(t-)=\Theta_{\infty} \cdot\left(1+R_{r} /\left(t-t_{s}(t-)-R_{a}\right)\right)$ where $\Theta_{\infty}=10 \mathrm{mV}, R_{a}=2 \mathrm{msec}, R_{r}=3 \mathrm{msec}$, and $s(t-)$ being the time of the latest spike before $t$. During the absolute refractory period $R_{a}$ after a spike, we have $\Theta=\infty$.

The neuron's excitatory (and inhibitory) synaptic inputs are generated by $n_{\mathrm{ex}}=5000$ (and $\left.n_{\mathrm{in}}=500\right)$ independent Poisson processes with rates $\lambda_{\mathrm{ex}}(t)=$ $L_{\mathrm{ex}} \cdot(1+a \cdot \cos (2 \pi f t))$ (and $\left.\lambda_{\mathrm{in}}=L_{\mathrm{in}}\right)$. We used $L_{\mathrm{ex}}=1 \mathrm{~Hz}, L_{\mathrm{in}}=2 \mathrm{~Hz}, a=0.5$ (or $a=0$ ) for the non-modulated regimes), $f=7 \mathrm{~Hz}$. For the simulations shown in Fig. 3 we adjusted synaptic strengths in order to obtain physiological ISIs. For the excitatory regimes we used $s_{\text {in }}=0$ and $s_{\text {ex }}=0.005-0.01$. For the balanced regimes we used $s_{\text {ex }}=0.5$ and $s_{\text {in }}=3-13$.

\section{References}

Abeles, M., Bergman, H., Gat, I., Meilijson, I., Seidemann, E., Tishby, N., Vaadia, E., 1995. Cortical activity flips among quasi stationary states. Proceedings of the National Academy of Science, USA 92, 8616-8620.

Arieli, A., Sterkin, A., Grinvald, A., Aertsen, A., 1996. Dynamics of ongoing activity: Explanation of the large variability in evoked cortical responses. Science 273, 1868-1871.

Bair, W., Koch, C., 1996. Temporal precision of spike trains in extrastriate cortex of the behaving macaque monkey. Neural Computation 8, 1185-1202.

Borst, A., Theunissen, F., 1999. Information theory and neural coding. Nature Neuroscience 2(11), 947-957.

Bugmann, G., Christodoulou, C., Taylor, J., 1997. Role of temporal integration and fluctuation detection in the highly irregular firing of a leaky integrator neuron with partial reset. Neural Computation 9, 985-1000.

Christodoulou, C., Bugmann, G., 2001. Coefficient of variation (CV) vs mean interspike interval (ISI) curves: what do they tell us about the brain? Neurocomputing 38-40, 1141-1149.

Eckhorn, R., 1999. Neural mechanisms of scene segmentation: recordings from the visual cortex suggest basic circuits for linking field models. IEEE Transactions on Neural Networks 10(3), 464-479.

Engel, A., Fries, P., Singer, W., 2001. Dynamic predictions: oscillations and synchrony in top-down processing. Nature Reviews Neuroscience 2(10), 704716. 
Feng, J., Brown, D., 2000. Impact of correlated inputs on the output of the integrate-and-fire model. Neural Computation 12, 671-692.

Hansel, D., Sompolinsky, H., 1996. Chaos and synchrony in a model of a hypercolumn in visual cortex. J. Comput. Neurosci. 3, 7-34.

Hebb, D., 1949. The organization of behavior. A neuropsychological theory. Wiley, New York.

Kenet, T., Bibitchkov, D., Tsodyks, M., Grinvald, A., Arieli, A., 2003. Spontaneously emerging cortical representations of visual attributes. Nature 425 , 954-956.

Knoblauch, A., 2003. Synchronization and pattern separation in spiking associative memory and visual cortical areas. PhD thesis, Department of Neural Information Processing, University of Ulm, Germany.

Knoblauch, A., Palm, G., 2001. Pattern separation and synchronization in spiking associative memories and visual areas. Neural Networks 14, 763780 .

Knoblauch, A., Palm, G., 2002. Scene segmentation by spike synchronization in reciprocally connected visual areas. I. Local effects of cortical feedback. Biological Cybernetics 87(3), 151-167.

La Camara, G., Fusi, S., Senn, W., Rauch, A., Lüscher, H.-R., 2002. When NMDA receptor conductances increase inter-spike interval variability. In: Dorronsoro, J. R. (Ed.), Artificial Neural Networks - ICANN 2002, International Conference, Madrid, Spain, August 28-30, 2002, Proceedings. Vol. 2415 of Lecture Notes in Computer Science. Springer, pp. 235-240.

Lin, J., Pawelzik, K., Ernst, U., Sejnowski, T., 1998. Irregular synchronous activity in stochastically-coupled networks of integrate-and-fire neurons. Network: Comput.Neural Syst. 9, 333-344.

Mainen, Z., Sejnowski, T., 1995. Reliability of spike timing in neocortical neurons. Science 268, 1503-1506.

Palm, G., 1982. Neural Assemblies. An Alternative Approach to Artificial Intelligence. Springer, Berlin.

Palm, G., 1990. Cell assemblies as a guideline for brain research. Concepts in Neuroscience 1, 133-148.

Palm, G., 1996. Information and surprise in brain theory. In: Rusch, G., Schmidt, S., Breidbach, O. (Eds.), Innere Repräsentationen - Neue Konzepte der Hirnforschung. DELFIN Jahrbuch. stw-Reihe, Suhrkamp, Frankfurt, pp. $153-173$.

Ringach, D., 2003. States of mind. Nature 425, 912-913.

Segundo, J., Vibert, J., Stiber, M., 1998. Periodically modulated inhibiton of pacemaker neurons. III. The heterogeneity of the postsynaptic spike trains and how control parameters affect it. Neuroscience 87, 15-47.

Shadlen, M., Newsome, W., 1994. Noise, neural codes and cortical organization. Curr. Opin. Neurobiol. 4, 569-579.

Shadlen, M., Newsome, W., 1998. The variable discharge of cortical neurons: Implications for connectivity, computation, and information coding. The Journal of Neuroscience 18(10), 3870-3896. 
Softky, W., 1995. Simple codes versus efficient codes. Current Opinion in Neurobiology 5, 239-247.

Softky, W., Koch, C., 1992. Cortical cells should fire regularly, but do not. Neural Computation 4, 643-646.

Softky, W., Koch, C., 1993. The highly irregular firing of cortical cells is inconsistent with temporal integration of random EPSPs. The Journal of Neuroscience $13(1)$, 334-350.

Stevens, C., Zador, A., 1998. Input synchrony and the irregular firing of cortical neurons. Nature Neuroscience 1(3), 210-217.

Troyer, T., Miller, K., 1997. Physiological gain leads to high ISI variability in a simple model of a cortical regular spiking cell. Neural Computation 9, 971-983.

Usher, M., Stemmler, M., Koch, C., Olami, Z., 1994. Network amplification of local fluctuations causes high spike rate variability, fractal firing patterns and oscillatory local field potentials. Neural Computation 6, 795-836. 